\title{
Sistema de inteligencia de negocio para la caracterización del turista de naturaleza
}

\section{Business intelligence system for the characterization of nature tourists}

Luis Efraín Velastegui López. ${ }^{1}$, Sayda Cecilia Chamba Melo. ${ }^{2}$, Wilson Wilfrido Quille

Chimborazo. ${ }^{3} \&$ Yasser Vázquez Alfonso. ${ }^{4}$

Recibido: 12-12-2019 / Revisado: 01-12-2019 / Aceptado: 06-01-2020 / Publicado: 07-02-2020

\section{Resumen}

DOI: https://doi.org/10.33262/concienciadigital.v3i1.1.1128

Los sistemas de inteligencia de negocio es una de las manifestaciones de la revolución científico técnica contemporánea ampliamente generalizada en países desarrollados. Actualmente los sistemas informáticos aplicados al turismo son muy usados en la práctica internacional ya que han evolucionado las metodologías de investigación turística, posibilitando solucionar los problemas de almacenamiento de datos cada vez más relacionado con el proceso de toma decisiones. En la investigación se realiza un análisis de los conceptos, herramientas y metodologías que se consideran importantes en el problema, reflejando el estado actual en la solución del mismo. El Ministerio de Turismo de Cotopaxi, al tener entre sus misiones y tareas el estricto control del turista que visita sus atractivos turísticos, posee un gran volumen de información que es generada diariamente. Como una respuesta a esa necesidad se decidió implementar un sistema de inteligencia de negocio, para el estudio del perfil de turista que visita los atractivos turísticos de Cotopaxi. La construcción e implementación de este sistema, utilizando el gestor de base datos Postgree SQL y la herramienta de modelado multidimensional Pentaho, presentó buena aceptación por parte de los directivos del turismo en Cotopaxi y contribuye a la toma de decisiones de los empresarios a nivel de atractivo turístico.

\footnotetext{
${ }^{1}$ Consorcio Ciencia Digital, Ambato, luisefrainvelastegui@ hotmail.com

${ }^{2}$ Instituto Superior Pedagógico Intercultural Bilingüe Ab. Martha Bucaram de Roldós, schamba@institutos.gob.ec

${ }^{3}$ Instituto Superior Pedagógico Intercultural Bilingüe Ab. Martha Bucaram de Roldós, wquille@ institutos.gob.ec

${ }^{4}$ Universidad de La Habana, yasser@ ftur.uh.cu
} 
Palabras claves: Turismo de naturaleza, sistema de inteligencia de negocio, Data Mart.

\section{Abstract}

The Business Intelligence Systems is one of the manifestations of the contemporary scientific technical revolution widely generalized in developed countries. Currently the computer systems applied to tourism are widely used in international practice since the methodologies of tourism research have evolved, making it possible to solve the problems of data storage increasingly related to the decision-making process. In the investigation, an analysis of the concepts, tools and methodologies that are considered important in the problem is realized, reflecting the current state in the solution of the same one. The Ministry of Tourism of Cotopaxi, having among its missions and tasks the strict control of the tourist who visits its tourist attractions, has a large volume of information that is generated daily. As a response to this need, it was decided to implement a Business Intelligence System to study the profile of tourists who visit the tourist attractions of Cotopaxi. The construction and implementation of this system, using the Postgree SQL Data Base Manager and the Multidimensional Pentaho modeling tool, presented good acceptance by the tourism managers in Pastaza and contributes to the decision making of the entrepreneurs at the level of attractiveness tourist.

Keywords: Nature tourism, Business Intelligence System, Data Mart.

\section{Introducción}

En la actualidad la actividad turística es una de las actividades comerciales más importantes del planeta. Para la Organización Mundial del Turismo (OMT, 2016) en los albores del nuevo milenio, el turismo se consolida como la principal actividad económica de muchos países y el sector de más rápido crecimiento en términos de ingresos de divisas y creación de empleo, generador de exportaciones del mundo y un factor importante en la balanza de pagos de muchos países. Según el secretario general de la OMT (2016a), el turismo mantiene la economía mundial "en movimiento", siendo la primera partida del comercio internacional, generando el mayor volumen de ingresos por exportaciones y dando empleo a millones de personas, producto de su carácter multi sectorial, y pese a las continuas turbulencias mundiales que amenazan su expansión y que se ha mantenido tenazmente, por ello muchos países se interesan seriamente en 
su desarrollo. Incluso, el turismo, en las últimas décadas ha configurado la geografía, demografía, economía y aspectos socio-culturales en muchas regiones del mundo (Morillo; 2007).

La información es muy importante para una empresa, así como lo es también que esté organizada. Actualmente contar con datos debidamente comprobados y un buen sistema de organización es vital para poder realizar estadísticas que pueden ayudar a la empresa a mejorar su desempeño no importa la actividad a la que se dedique (Escobar; 2007).

Ecuador tiene posición privilegiada para desarrollar el turismo sostenible por su diversidad formando parte de los 17 países mega diversos, con un alto potencial para posicionarse como destino natural ya que cuenta con la mayor diversidad de vida por unidad de superficie en la escala mundial, haciéndose énfasis en el slogan Ecuador "ama la vida" y en el concepto de turismo consciente. Cotopaxi es una provincia interandina que cuenta con gran cantidad de atractivos turísticos tanto recursos naturales como ríos, bosques primarios, montañas, flora y fauna endémica y manifestaciones culturales como leyendas, costumbres variedad y diversidad de nacionalidades, 7 de las 14 nacionalidades del país se encuentran en esta provincia, además es la más grande del país (Acosta; 2014).

El Ministerio del Turismo conformó una asociación con los propietarios de varios atractivos y escenarios turísticos implementados como una inversión privada y que particularmente unos con otros estén diferenciados, lo que ha permitido consolidar una oferta variada, actualmente son los sitios más visitados de Cotopaxi y de mayor accesibilidad al público, por lo que requieren de una asistencia técnica para determinar el segmento de mercado del visitante que son frecuentados en estos atractivos turísticos y un trabajo conjunto que permita mejorar la calidad del servicio, por lo que en esta investigación se propone desarrollar un sistema de inteligencia de negocio para el estudio del perfil de turista que visita los atractivos turísticos de Cotopaxi.

\section{Desarrollo}

La presente investigación se realiza en el año 2017 en los atractivos turísticos de la provincia de Cotopaxi que se encuentra ubicada en la región de la sierra ecuatoriana.

Para el desarrollo del sistema de inteligencia de negocios (SIN), se utiliza la metodología de desarrollo de Kimball (2004), ya que este sistema tiene como propósito facilitar el entendimiento de la información turística, proveniente de los atractivos turísticos que se encuentran en la 
provincia de Cotopaxi.

La metodología se basa en lo que Kimball denomina ciclo de vida dimensional del negocio (Business Dimensional Lifecycle). Este ciclo de vida del proyecto de DW, está basado en cuatro principios básicos según (Morrow, 2014):

1. Centrarse en el negocio.

2. Construir una infraestructura de información adecuada.

3. Realizar entregas en incrementos significativos: (este principio consiste en crear el almacén de datos DW en incrementos entregables en plazos de seis a doce meses. En este punto, la metodología se parece a las metodologías ágiles de construcción de software).

4. Ofrecer la solución completa. En este se punto proporcionan todos los elementos necesarios para entregar valor a los usuarios de negocios, para esto ya se debe tener un almacén de datos bien diseñado, se deberán entregar herramientas de consulta ad hoc, aplicaciones para informes, análisis avanzado, capacitación, soporte, sitio web y documentación.

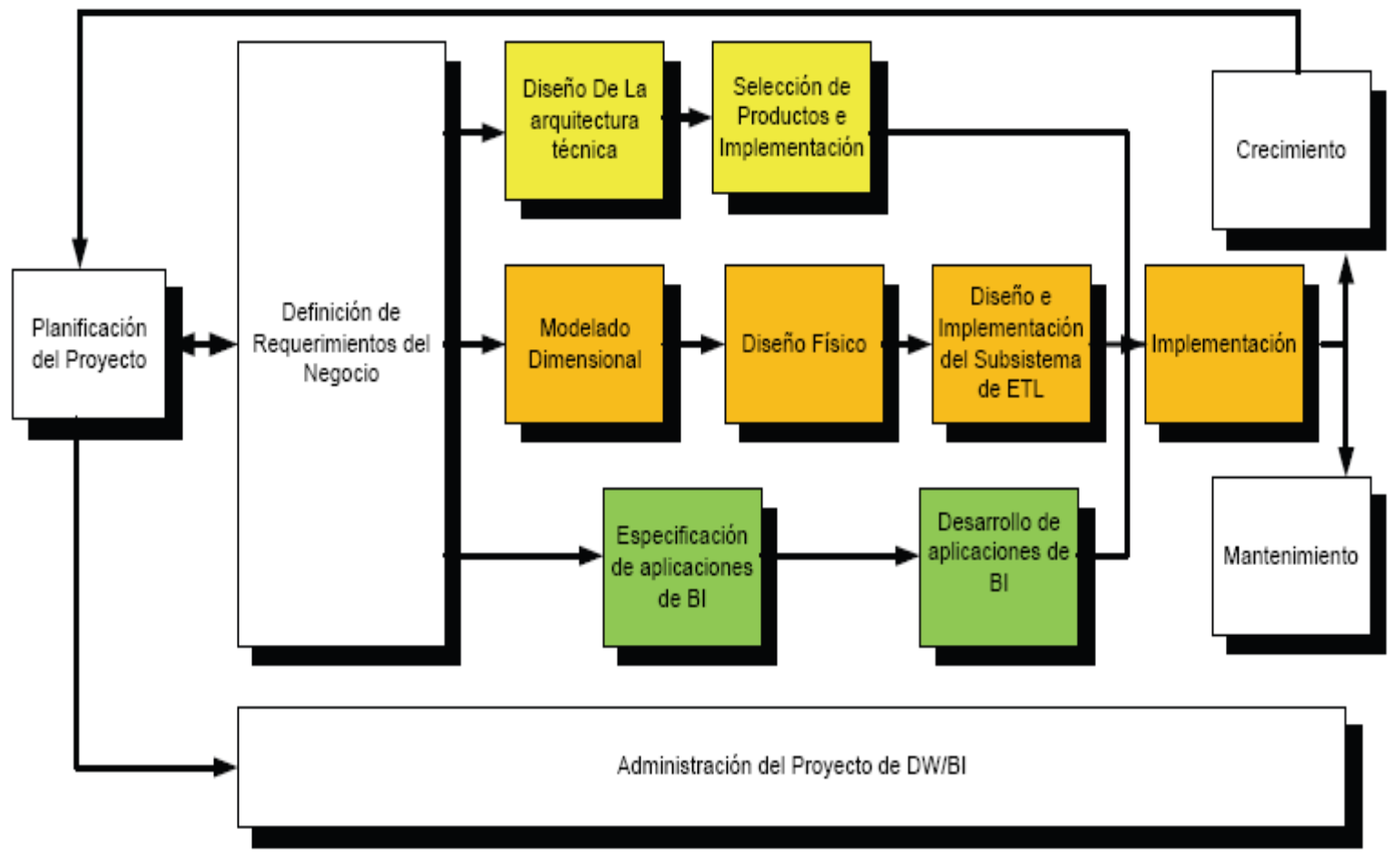

Figura 1: Ciclo de vida, metodología Kimball.

Fuente: Morrow (2014) 
En la figura 1 se presenta el ciclo de vida de la metodología de Kimball que consiste en:

Planificación del proyecto: En este proceso se determina el propósito del proyecto de DW/BI, sus objetivos específicos y el alcance del mismo, los principales riesgos y una aproximación inicial a las necesidades de información.

Definición de requerimientos del negocio: La definición de requerimientos, es un proceso en el que se entrevista al personal de negocio y técnico, aunque siempre conviene, tener un poco de preparación previa. En esta tarea, se debe aprender sobre el negocio, los competidores, la industria y los clientes del mismo. Se debe dar una revisión a todos los informes posibles de la organización; rastrear los documentos de estrategia interna; entrevistar a los empleados, analizar lo que se dice en la prensa acerca de la organización, la competencia y la industria y se deben conocer los términos y la terminología del negocio.

Modelado dimensional: Es un proceso dinámico y altamente iterativo. Comienza con un modelo dimensional de alto nivel obtenido a partir de los procesos priorizados y descritos en la tarea anterior.

Diseño físico: En esta tarea, se contestan las siguientes preguntas:

- ¿Cómo puede determinar cuán grande será el sistema de DW/BI?

- ¿Cuáles son los factores de uso que llevarán a una configuración más grande y más compleja?

- ¿Cómo se debe configurar el sistema?

- ¿Cuánta memoria y servidores se necesitan? ¿Qué tipo de almacenamiento y procesadores?

- ¿Cómo instalar el software en los servidores de desarrollo, prueba y producción?

- ¿Qué necesitan instalar los diferentes miembros del equipo de DW/BI en sus estaciones de trabajo?

- ¿Cómo convertir el modelo de datos lógico en un modelo de datos físicos en la base de datos relacional?

- ¿Cómo conseguir un plan de indexación inicial?

- ¿Debe usarse la partición en las tablas relacionales?

Diseño e implementación del subsistema de extracción, transformación y carga (ETC): El subsistema ETC es la base sobre la cual se alimenta el DW. Si se diseña adecuadamente, puede extraer los datos de los sistemas de origen de datos, aplicar diferentes reglas para aumentar la 
calidad y consistencia de los mismos, consolidar la información proveniente de distintos sistemas, y finalmente cargar (grabar) la información en el DW en un formato acorde para la utilización por parte de las herramientas de análisis.

Implementación: La implementación representa la convergencia de la tecnología, los datos y las aplicaciones de usuarios finales accesible desde el escritorio del usuario del negocio. Existen varios factores extras que aseguran el correcto funcionamiento de todas estas piezas, entre ellos se encuentran la capacitación, el soporte técnico y la comunicación.

Mantenimiento y crecimiento del DW: Para administrar el entorno del DW existente es importante enfocarse en los usuarios de negocio, los cuales son el motivo de su existencia, además de gestionar adecuadamente las operaciones del DW, medir y proyectar su éxito y comunicarse constantemente con los usuarios para establecer un flujo de retroalimentación. En esto consiste el mantenimiento. Finalmente, es importante sentar las bases para el crecimiento y evolución del DW en donde el aspecto clave es manejar el crecimiento y evolución de forma iterativa utilizando el ciclo de vida propuesto, y establecer las oportunidades de crecimiento y evolución en orden por nivel de prioridad.

Para definir los requerimientos del negocio se hace necesario realizar entrevistas a los directivos y empresarios, para concretar las funciones del SIN que se actualizará en los diferentes atractivos turísticos.

Requerimientos generales: En el departamento del Ministerio de Turismo de Cotopaxi se registra un gran conjunto de información generada diariamente, proveniente de los diferentes atractivos turísticos que se encuentran en la provincia de Cotopaxi. Al implementarse el SIN se sustituirá una versión anterior de una base datos, que posibilitará centralizar y organizar la información, logrando así, que su búsqueda sea más fácil y que se pueda tener una mejor visualización de los datos. El software constará con herramientas como gráficos y reportes que no se encontraban en el sistema informático anterior y son de gran importancia para facilitar tareas al directivo responsable de la toma de decisiones.

Requerimientos arquitectónicos: Los requerimientos arquitectónicos son herramientas informáticas necesarias para el desarrollo del SIN que requiere:

- Un SGBD para almacenar la información que se generará del proceso de ETC. Se organizará 
la información en esquemas, el esquema pntemp contendrá las tablas históricas y por otra parte en el esquema at tur se almacenarán las tablas de hechos y de dimensiones.

- El sistema podrá ser utilizado bajo los sistemas operativos Windows o Linux.

- Se utilizará Kettle Pentaho para realizar el proceso de ETC, en dicho proceso se capturará toda la información de diferentes fuentes logrando una centralización de la información.

- Se utiliza Pentaho BI Server, pues en él se implementará el sistema y sus funcionalidades.

- Se debe utilizar Java JDK 1.5 o una versión superior ya que la mayoría de las herramientas a utilizar están implementadas en Java.

Requerimientos de desarrollo: Para el desarrollo del SIN los requerimientos se dividen en dos grupos:

- Hardware, donde se necesita una computadora con los siguientes requisitos mínimos:

> Procesador $1.50 \mathrm{GHz}$.

$1 \mathrm{~GB}$ de RAM.

D Espacio en disco duro $20 \mathrm{~Gb}$.

$>$ Lector de DVD.

- Software.

$>$ Sistema Operativo Windows o Linux.

$>$ Navegador web.

$>$ Máquina virtual de Java.

$>$ Plataforma de Pentaho.

El sistema se implementó en ambiente web sobre el servidor de inteligencia de negocio pentaho BI Server. La primera interfaz que muestra el sistema es donde el usuario tendrá que escribir su usuario y contraseña, insertados previamente por el administrador del sistema. Esta interfaz es generada por el servidor, y a diferencia del software anterior cada usuario posee contraseña propia.

A continuación, se muestra la interfaz de bienvenida al sistema de la SIGEMP, en el mismo se inserta nombre del usuario y contraseña dando acceso a la página principal creada por la empresa. 


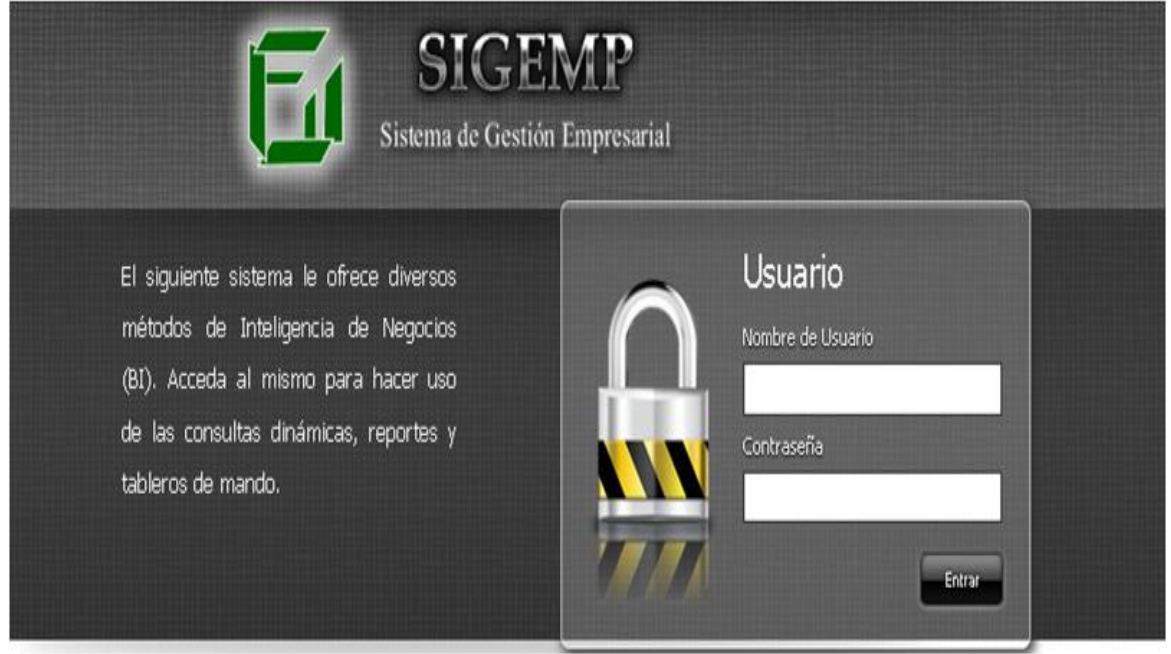

Figura 2: Página de autenticarse del sistema

Una vez que el usuario se autentifique con su nombre y contraseña va a encontrarse con la página principal que se muestra a continuación, se selecciona la pestaña de Atractivos que dará inicio al SIN y ahí se muestran todas las funcionalidades que se implantaron (Figura 3).

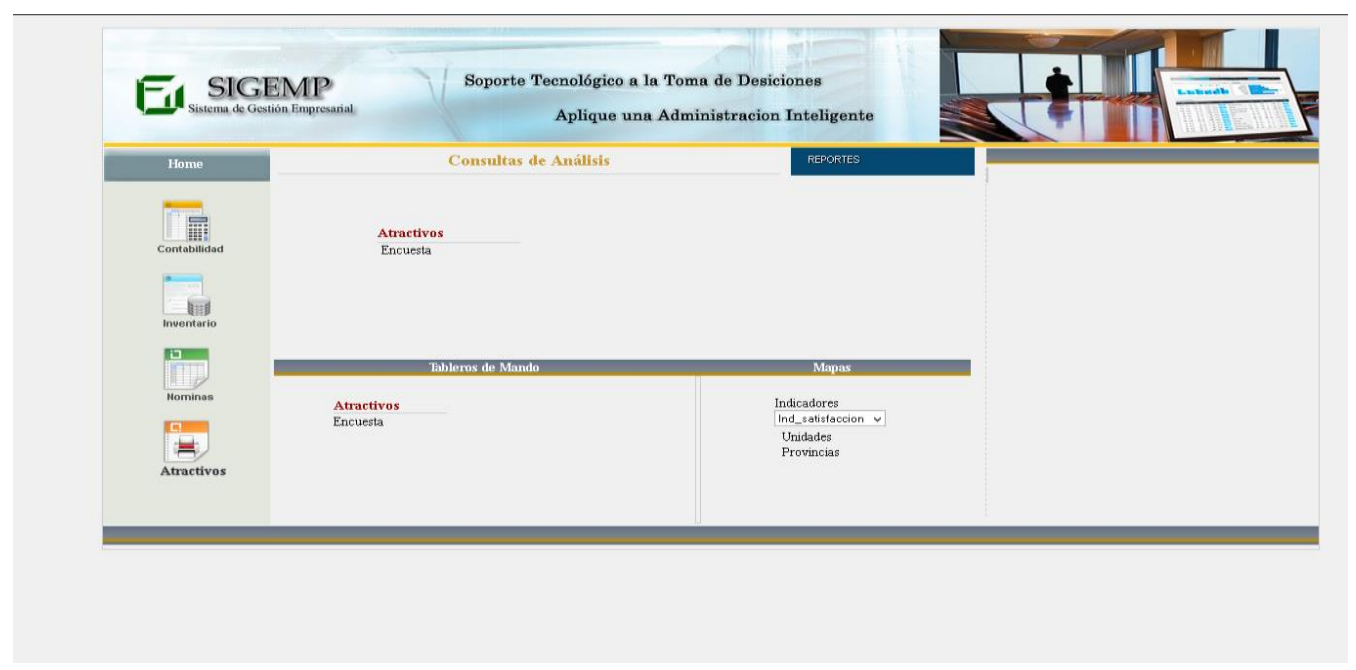

Figura 3: Página principal del SIN.

Fuente: Elaboración propia.

En la figura 4 se muestra la salida del análisis estadístico descriptivo realizado a las diferentes variables que componen la encuesta realizada a los turistas que visitaron los diferentes atractivos turísticos de la provincia Cotopaxi. 


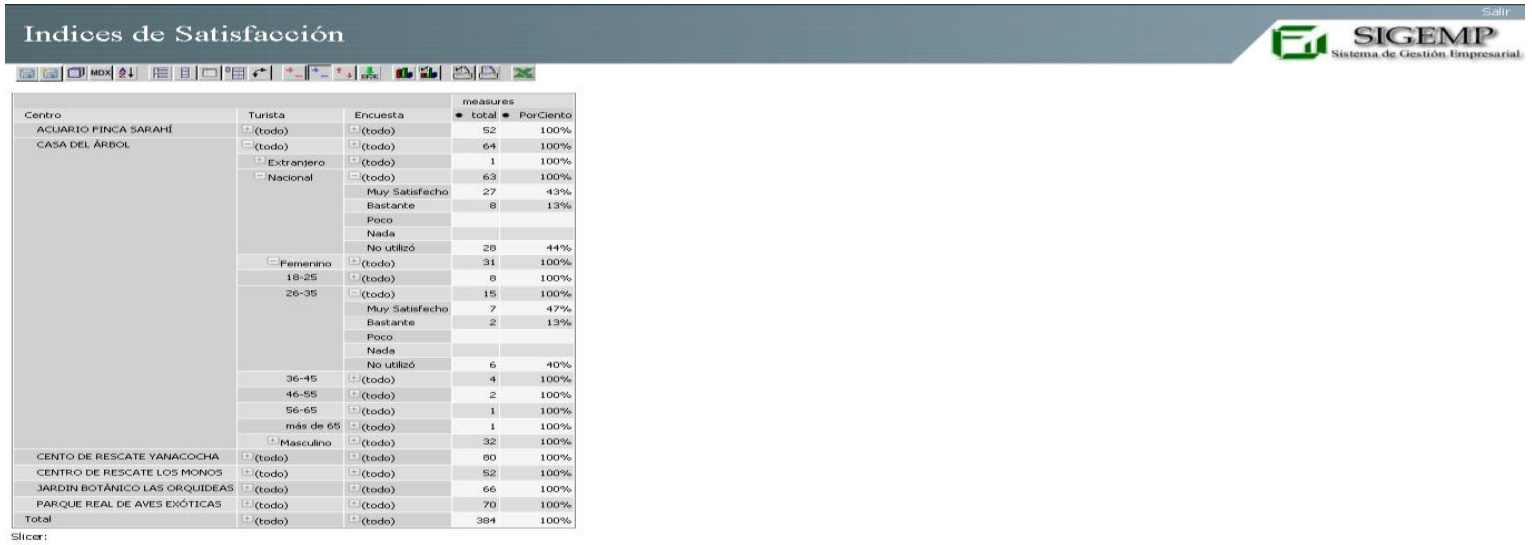

Figura 4: Resultados del procesamiento de las encuestas por el SIN.

Fuente: Elaboración propia.

Otro resultado en este sistema es el que se ilustra en la figura 5, donde se observa el comportamiento de las variables que componen la encuesta realizada a los turistas que visitan los atractivos turísticos de Cotopaxi. El rango de edades de los turistas que más visita estos atractivos es (26-35), el sexo que más predomina entre los turistas visitantes es el femenino (53\%) y la gran mayoría de los turistas encuesta es de procedencia nacional. La gráfica de línea muestra el comportamiento de la satisfacción de los turistas en los diferentes atractivos turístico de los diferentes centros turísticos de Cotopaxi, reflejando que el mayor porciento lo obtuvo la Laguna del Quilotoa.

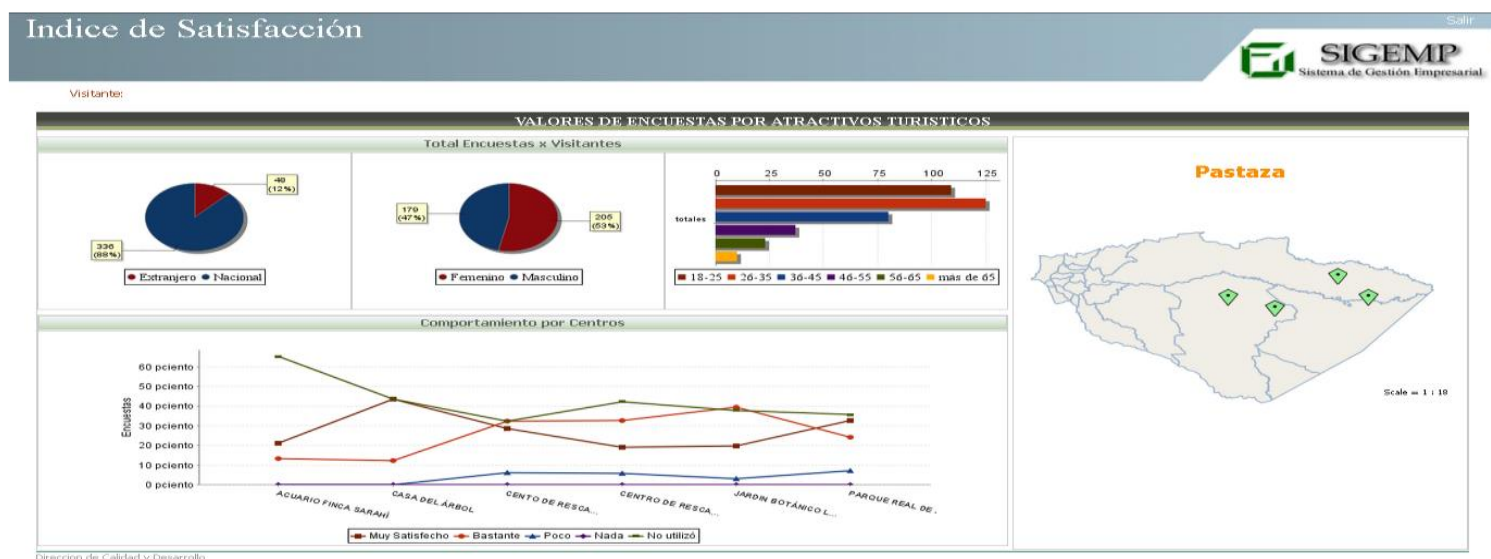

Figura 5: Resultados del procesamiento de las encuestas por el SIN.

Fuente: Elaboración propia. 
Estos resultados complementan las investigaciones realizadas en el campo del estudio de la satisfacción del turista de naturaleza como son: Escobar (2007), Font (2010), Reverter y Plaza (2011) y Acosta (2014), las cuales fueron un punto de partida para esta investigación.

\section{Conclusiones}

Se seleccionaron los métodos y herramientas de desarrollo adecuados que permitieron el diseño de un sistema de inteligencia de negocio para el turismo de naturaleza con calidad, de acuerdo a los requerimientos de usuarios.

Se diseñaron las bases de datos relacionadas con la información hidrológica proveniente de los procesos de ETC utilizando los gestores de base de datos, lográndose el almacenamiento de una cantidad mínima de datos históricos procedentes de los diferentes atractivos turísticos a través de los años.

Se implementó un sistema de inteligencia de negocio para el análisis de la información turística enfocado en los atractivos turísticos de los diferentes centros turísticos de Cotopaxi, incorporándose herramientas gráficas y de panel.

\section{Referencias Bibliográficas}

Acosta, A. (2014). Perfil del turista que visita los emprendimientos de la asociación de atractivos turísticos privados de Cotopaxi, para la promoción turística del cantón en base a la segmentación del mercado en temporada alta 2013. Tesis previa a la obtención del título de Ingeniería en Turismo. Universidad de Cotopaxi. Ecuador. p.280.

Escobar, M. (2007). Desarrollo de datawarehouse de información turística del Ecuador. Tesis presentada en opción al Título de Ingeniero en Sistema e Informática. Escuela Politécnica del Ejército, Sangolqui, Ecuador. 102p.

Font, M. (2010). Reflexiones acerca del turismo de naturaleza. Centro de Estudios de la Universidad de Matanzas. Cuba. 114p.

Kimball, R. (2004). The Data Warehouse ETL Toolkit. Wiley Publishing, Inc. 120p.

Moriillo, M. (2007). Análisis de la calidad del servicio hotelero mediante la escala de SERVQUAL. Revista Visión Gerencial, ISSN 1317-8822, p269-297. julio - diciembre.

Morrow, J. (2014). Inteligencia de Negocio. Metodología de Kimball. 17p. http://inteligenciadenegociosval.blogspot.com/2014/01/metodologia-de-kimball.html 
[consultado 27/07/16].

OMT (2016). WTO World tourism barometer: international tourism on track for another strong year. Dpto. de Prensa y Comunicaciones de la OMT.

http://www.unwto.org/newsroom/releases/2005/octuber/barometer.html [consultado 27/07/16].

OMT (2016): To be fourth year of sustained growth. Dpto. de Prensa y Comunicaciones.

http://www.unwto.org/newsroom/releases/2006/november/barometer06.htm[consultado 27/07/16].

Reverter-Masia; Plaza-Montero, D. (2011): Ocio, Deporte y Turismo: situación actual y líneas de negocio. Deporte y sociedad. http://www.uff.br/esportesociedade/pdf/es1603.pdf. [consultado 27/07/16].

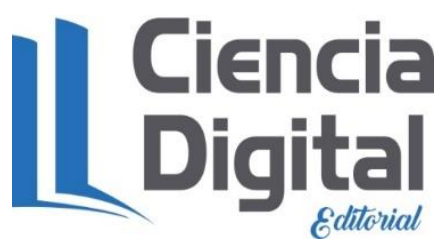




\section{Para citar el artículo indexado}

Velasteguí López, L. E., Chamba Melo, S. C., Quille Chimborazo, W. W., \& Vázquez Alfonso, Y. (2020). Sistema de inteligencia de negocio para la caracterización del turista de naturaleza. ConcienciaDigital, 3(1.1), 5-16. https://doi.org/10.33262/concienciadigital.v3i1.1.1128

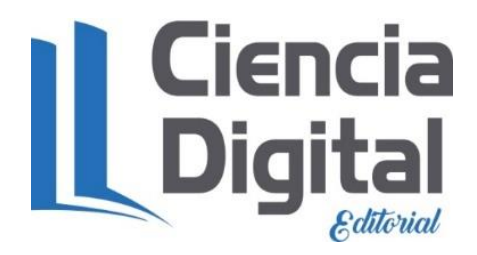

El artículo que se publica es de exclusiva responsabilidad de los autores y no necesariamente reflejan el pensamiento de la Revista Conciencia Digital.

El articulo queda en propiedad de la revista y, por tanto, su publicación parcial y/o total en otro medio tiene que ser autorizado por el director de la Revista Conciencia Digital.
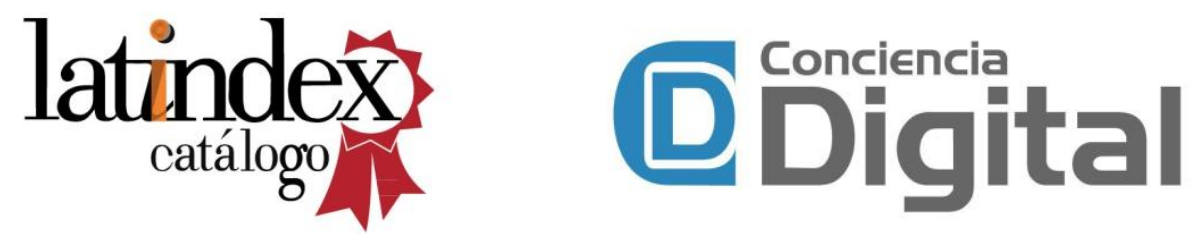